\title{
Lau, Thomas/Reinhardt, Volker/Voigt, Rüdiger (Hrsg.): Edmund Burke. Vater des Konservatismus? (Staatsverständnisse, Bd. 150), 255 S., Nomos, Baden-Baden 2021.
}

\author{
André Kahl
}

Angenommen: 17. Dezember 2021 / Online publiziert: 22. Februar 2022

(C) Der/die Autor(en) 2022

Die verschiedenen Verständnisse des Staates in seinen neuzeitlichen Ausprägungen darzustellen, ist das erklärte Ziel der Reihe „Staatsverständnisse“. Als einer der bedeutendsten Redner der Whigs und Bezugspunkt einer ganzen Weltanschauung hat Edmund Burke das Staatsdenken maßgeblich beeinflusst, weshalb sich die leitende Frage des Bandes nach der, ,Wirkungsgeschichte dieses irisch-britischen Schriftstellers“ (S. 14) geradezu aufdrängt. Thematisch untergliedert sich der Sammelband in vier Abschnitte. Im ersten Teil widmen sich die Autor_innen dem Verhältnis von Burke zur Französischen Revolution. Volker Reinhardt schreibt in seinem Beitrag gegen die populäre Deutung Burkes als Propheten des Terrors an. Die vergleichsweise früh im Revolutionsverlauf erschienenen „Reflections“ basieren lediglich auf einer knappen „historische[n] Ereigniskette von etwa sechzehn Monaten“ (S. 20). Entsprechend habe es kaum Anhaltspunkte für die nachfolgende Eskalationsspirale zwischen 1792 und 1794 gegeben. Dem synchronen Zugang folgen zwei diachron angelegte Beiträge, die Burkes Bezüge zur Französischen Revolution in den Kontext der englischen Revolutionen des 17. Jahrhunderts stellen. Beide Artikel ergänzen sich ausnehmend gut, denn während Ulrich Niggemann verstärkt die Revolutionsnarrative der glorious revolution in den Blick nimmt, stehen bei Skadi Siiri Krause die Diskurse und radikalen Forderungen im Zuge der Englischen Bürgerkriege (1639-1651) im Fokus. Gegenstand des Aufsatzes von Thomas Lau ist Burkes Nationenkonzept. Dieses besitzt keinen „Ewigkeitscharakter“ (S. 93), sondern ist sterblich und wird durch Bedingungsgrößen wie Atheismus und Intoleranz zu Grabe getragen.

Die Frage, ob Burke angemessen als „Ahnherr des Konservatismus“ (S. 111) verstanden werden kann, ist leitend für den zweiten thematischen Block des Sammelbandes. Entgegen einigen prominenten Vordatierern des Konservatismus - zu

André Kahl $(\bowtie)$

Martin-Luther-Universität Halle-Wittenberg, Halle an der Saale, Deutschland

E-Mail: andre.kahl@politik.uni-halle.de 
nennen sind Panajotis Kondylis und Klaus Epstein - spricht für Henning Ottmann „viel dafür, den [...] Konservatismus mit dem Epochenereignis der Französischen Revolution und der Reaktion auf diese zu verbinden“ (S. 114). Insofern sei die Etikettierung Burkes als Konservativer zeitlich passend. Der Präferenz für das Konkrete folgend sei er kaum an „Globaltheorien“ (S. 126) interessiert gewesen, wobei es ,,[i]hm genügte [...], das geschichtlich Bewährte zu erhalten und es nach Möglichkeit zu verbessern“ (ebd.). Daneben stütze sich sein Konservatismus auf „eine Lehre von der prescription und eine Rehabilitierung des Vorurteils“" (S. 123). In ihrem zweiten Beitrag empfiehlt Skadi Siiri Krause eine stärkere Kontextualisierung zur Beantwortung der kapitelleitenden Frage. Dann nämlich werde deutlich, dass das Wirken des Iren in eine Zeit der Ausdifferenzierung ,innerhalb des liberalen Lagers“ (S. 129) fällt. Neben einer ,,antiabsolutistische[n], verfassungsrechtliche[n] Denkrichtung“ (S. 130), welcher Burke zuzuordnen sei, gab es eine ,radikale, demokratisch geprägte Strömung“ (S. 131). In diesem Deutungskampf sei der Gradualist gleichsam zum Konservativen gemacht worden, weil viele seiner politischen Standpunkte nicht mehr dem ,Zeitgeist“ (S. 145) entsprächen. Auch Michael Becker hält die häufig pejorative Zuschreibung des Konservativen für vereinseitigend. Oftmals gehe damit die Unterstellung fehlender Überzeugungen einher, was besonders mit Blick auf Burkes koloniale Positionen zurückgewiesen werden kann. Ein derartiger Zuschnitt offenbare nicht nur einen ,,vehemente[n] Verteidiger der Freiheit“" (S. 169), mehr noch, Burke warte gar mit einem „Postkolonialismus avant la lettre“ (S. 151) auf.

Den dritten Themenblock des Bandes bilden verschiedene Sichtweisen der modernen Ideengeschichte auf Burke. Norbert Campagna stellt in seinem Beitrag interessante Parallelen zwischen Burke und Alexis de Tocqueville heraus. Der Dissens über den Status der Französischen Revolution - Burke glaubt an die „Neubelebung der französischen Verfassung“ (S. 178), während der einstmalige Außenminister Frankreichs jene Umwälzungen als offenkundige Vorhut eines neuen Zeitalters der Gleichheit bewertet - dürfe nicht über manifeste Konvergenzpunkte hinwegtäuschen. Beiden sei gemein, dass sie die mit Vorurteilen einhergehende Erwartungssicherheit preisen, wohingegen sie die Gefahren einer ungehinderten Volkssouveränität für ausgemacht halten. Oliver Hidalgo stellt in seinem Beitrag heraus, dass das von Thomas Paine mit-geprägte Konzept der repräsentativen Demokratie kaum ohne den „Stichwortgeber“ (S. 201) Burke denkbar gewesen wäre. Indem dieser vollmundig die Unvereinbarkeit von Repräsentation und Demokratie behauptete, habe er Paine geradewegs dazu provoziert, den Möglichkeiten-Horizont einer solchen Amalgamierung auszuloten. Als ,eine[n] der erfolgreichsten Geschichtenerzähler des britischen Parlamentarismus“ (S. 216) wird Burke von Jürgen Kamm beschrieben. Was politische Akteure heute vielfach wissen und durch die Zuhilfenahme von allerlei PRExperten wie Spin-Doktoren zu professionalisieren suchen, sei bereits von Burke erkannt worden: ,es geht darum, den Wählerinnen und Wählern den eigenen politischen Gestaltungswillen in Form einer plausiblen, nachvollziehbaren Geschichte näher zu bringen“" (S. 215).

Narrativ wird die englische Verfassungsgeschichte als „Heldenepos“ (S. 228) beschrieben, wohingegen die Darstellung des französischen Revolutionsverlaufs bisweilen zwischen Satire und Tragödie changiert. Der abschließende Beitrag von 
Rüdiger Voigt bildet gleichzeitig den mit „Parlamentarismus und freies Mandat“ überschriebenen letzten Themenblock, wobei der Autor zentrale Schwerpunkte von Burkes Denken pointiert. $\mathrm{Zu}$ nennen sind etwa sein Verständnis des Staates als „geronnene historische Vernunft“ (S. 245) oder seine „merkwürdige, wenn nicht reaktionäre Demokratievorstellung“ (S. 246). Leider wurde der multiperspektivische Zuschnitt der vorangehenden drei Kapitel hier nicht weitergeführt, was den rundum gelungenen Sammelband etwas abrupt zu einem Ende kommen lässt.

Funding Open Access funding enabled and organized by Projekt DEAL.

Open Access Dieser Artikel wird unter der Creative Commons Namensnennung 4.0 International Lizenz veröffentlicht, welche die Nutzung, Vervielfältigung, Bearbeitung, Verbreitung und Wiedergabe in jeglichem Medium und Format erlaubt, sofern Sie den/die ursprünglichen Autor(en) und die Quelle ordnungsgemäß nennen, einen Link zur Creative Commons Lizenz beifügen und angeben, ob Änderungen vorgenommen wurden.

Die in diesem Artikel enthaltenen Bilder und sonstiges Drittmaterial unterliegen ebenfalls der genannten Creative Commons Lizenz, sofern sich aus der Abbildungslegende nichts anderes ergibt. Sofern das betreffende Material nicht unter der genannten Creative Commons Lizenz steht und die betreffende Handlung nicht nach gesetzlichen Vorschriften erlaubt ist, ist für die oben aufgeführten Weiterverwendungen des Materials die Einwilligung des jeweiligen Rechteinhabers einzuholen.

Weitere Details zur Lizenz entnehmen Sie bitte der Lizenzinformation auf http://creativecommons.org/ licenses/by/4.0/deed.de.

\section{Kroll, Thomas/Severin-Barboutie, Bettina (Hrsg.): Wider den Kapitalismus. Antikapitalismen in der Moderne, 294 S., Campus, Frankfurt a.M. u.a. 2021.}

\section{Anette Schlimm}

Angenommen: 17. Dezember 2021 / Online publiziert: 24. Januar 2022

(C) Der/die Autor(en) 2022

Er war eine Irritation für die bundesdeutsche Gesellschaft und löste einen veritablen Skandal aus: Hannsheinz Porst war Millionär und Mitglied in der FDP - gleichzeitig aber auch in der SED. 1969 wurde er für seine Zusammenarbeit mit dem Ministerium für Staatssicherheit zu 33 Monaten Gefängnis verurteilt. War er nun ein Kapitalist

Anette Schlimm $(\bowtie)$

Ludwig-Maximilians-Universität, München, Deutschland

E-Mail: anette.schlimm@1rz.uni-muenchen.de 